\title{
ELECTRON HEAT CONDUCTION IN ICF TARGETS
}

Juan R. Sanmartín

\section{TABLE OF CONTENTS}

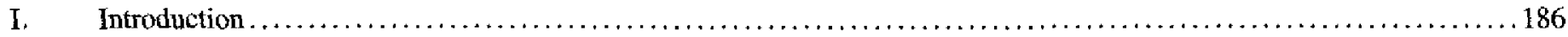

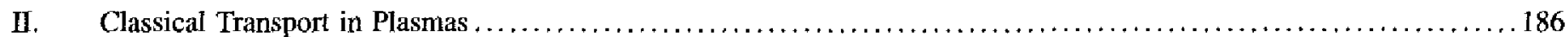

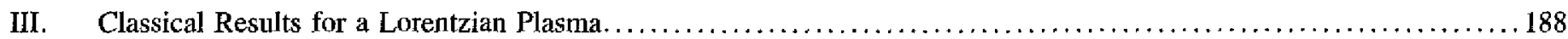

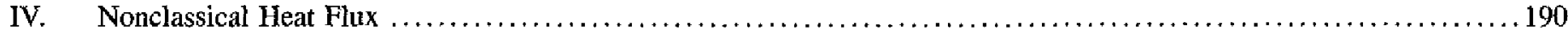

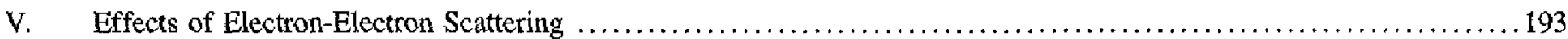

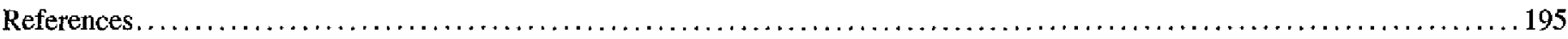




\section{INTRODUC'TION}

As noted in foregoing chapters of this book, electron heat transport is a crucial part of the physics in direct-drive laser fusion, that is, in targets that do not produce a copious X-ray population. Since the plasma ablated from a target is flowing outward, heat conduction is the only mechanism capable of carrying the energy (absorbed at densities up to critical) to the larger densities prevailing at the ablation surface. There is also outward conduction to the low-density, faraway plasma, and it is again of interest to determine how much heat is directly lost to the expansion flow. Independently, thermal diffusion appears to play a key role in the smoothing of unavoidable nonuniformities in the absorption of laser energy that drives the expansion.

In the following, we shall first discuss the similarities and differences between transport processes in plasmas and in neutral gas mixtures; this will help in clarifying approaches to the kinetics of plasma transport that can be found in related literature. Secondly, we describe the classical calculation of heat conduction in a Lorentzian plasma, fully including magnetic field and electric current effects, which are certain to appear in geometties that are not strictly one-dimensional. Next, we review the difficulties that have been found when using classical results to interpret laser experiments, and the various onedimensional models introduced in the past to cope with such difficulties; in particular, we discuss, in some detail, nonlocal models that calculate the thermal conduction as a convolution on formulae describing transport as local. Finally, we discuss the extension of those analyses to non-Lorentzian plasmas.

\section{CLASSICAL TRANSPORT IN PLASMAS}

In a mixture of two neutral gases, where local equilibrium prevails and distribution functions are nearly Maxwellian, temperatures and mean directed velocities are nearly the same for either species. Naturally, particle number densities may be arbitrarily different. The small relative velocity between species is then important only in the sense that it will induce changes of concentration throughout the system, that is, for diffusion.

A plasma behaves just the opposite in this respect. Standard transport theory is applicable when the characteristic macroscopic length, $\mathrm{H}$, is larger than the mean free path for collisions that lead to a Maxwellian distribution. In the usual quasi-ideal plasma that has a large number of particles in a Debye sphere, the Debye length is short when compared with all mean free paths; thus, transport processes are basically quasi-neutral, the density ratio being a constant $n_{v} / n_{i}=$ ion charge state $Z$. On the other hand, and because the electron-to-ion mass ratio $\mathrm{m}_{\mathrm{e}}$ $\mathrm{m}_{\mathrm{i}}$ is very small, energy equipartition need not be established in a distance $\mathrm{H}$; hence, for generality, one should allow different temperatures, i.e., $T_{e} \neq T_{i}$, the use of a single one unduly restricting the analysis. Also, one can easily verify that the difference between ion and electron velocities, $\bar{u} \equiv$ $\overline{\mathrm{u}}_{\mathrm{e}}-\overline{\mathrm{u}}_{\mathrm{j}}$, may be as large as $\overline{\mathrm{u}}_{\mathrm{e}}$ or $\overline{\mathrm{u}}_{\mathrm{i}}$. Because of both quasineutrality and low mass ratio, one generally has both $u_{e}$ and $\mathrm{u}_{\mathrm{i}} \ll\left(\mathrm{T}_{\mathrm{e}} / \mathrm{m}_{\mathrm{e}}\right)^{1 / 2}$; for instance, in an expansion into a vacuum, as in laser fusion, characteristic velocities are of the order $\left(\mathrm{ZT}_{\mathrm{e}}\right.$ $\left.+T_{i}\right)^{1 / 2} / m_{i}^{1 / 2}$. If $\lambda_{\mathrm{r}}$ is the mean free path for scattering of thermal electrons by ions, the friction between species requires that $\mathrm{u}$ be, at most, of the order of $\left(\mathrm{T}_{\mathrm{e}} / \mathrm{m}_{\mathrm{e}}\right)^{1 / 2} \lambda_{\mathrm{T}} / \mathrm{H}$, and therefore comparable, in principle, to $u_{i}$ or $u_{e}$.

The literature on the determination of particular, or complete sets of, transport coefficients in a plasma is extensive. A variety of computational methods have been used, with collision terms of both Boltzmann and Fokker-Planck type. We will not review that work here. The reader should consult Shkarofsky et al., ${ }^{2}$ Hochstim and Massel, ${ }^{3}$ or Epperlein and Haines; ${ }^{4}$ one should also sec Chapman and Cowling. ${ }^{5}$ We shall just briefly mention some of the earliest published work. A direct numerical calculation of the departure of the distribution function f from a local Maxwellian $\mathrm{f}_{\mathrm{M}}$ was first performed by Spitzer and Härm ${ }^{6}$ and, recently, in Reference 4. Grad's 13moment method was used by Burgers ${ }^{7}$ and Herdan and Liley. ${ }^{8}$ A variational method was introduced by Marshall (cited in Reference 9) and Robinson and Bernstein. ${ }^{10}$ Most calculations, beginning with early papers by Cowling ${ }^{11}$ and Landshoff ${ }^{12}$ have involved a Laguerre expansion of the departure $f-f_{M}$.

The first systematic analysis of transport that considered arbitrary differences between ion and electron temperatures and velocities was carried out by Braginskii. ${ }^{13}$ We shall use his approach, though we avoid the Laguerre expansion he used, restricting our exposition to analytical results as far as possible. Errors in Braginskii's numerical results have been detected recently by Epperlein ${ }^{34}$ and Epperlein and Haines ${ }^{4}$ (see also work by Lee and More $^{15}$ ).

Thus, following Braginskii, we consider separate momentum and entropy (instead of energy) equations for each species:

$$
\begin{gathered}
\mathrm{m}_{\alpha} \mathrm{n}_{\alpha}\left(\frac{\partial}{\partial \mathrm{t}}+\overline{\mathrm{u}}_{\alpha} \cdot \nabla\right) \overline{\mathrm{u}}_{\alpha}=-\nabla\left(\mathrm{n}_{\alpha} \mathrm{T} \alpha\right)-\nabla \cdot \overline{\bar{\Pi}}_{\alpha}+ \\
+\mathrm{n}_{\alpha} \mathbf{e}_{\alpha}\left(\overline{\mathrm{E}}+\overline{\mathrm{u}}_{\alpha} \alpha \overline{\mathrm{B}}\right)+\overline{\mathrm{R}}_{\alpha} \\
\mathrm{n}_{\alpha} \mathrm{T}_{\alpha}\left(\frac{\partial}{\partial \mathrm{t}}+\overline{\mathrm{u}}_{\alpha} \cdot \nabla\right) \ln \left(\mathrm{T}_{\alpha}^{3 / 2} / \mathrm{n}_{\alpha}\right) \\
=-\nabla \cdot \overline{\mathrm{q}}_{\alpha}-\bar{\Pi}_{\alpha}: \nabla \overline{\mathrm{u}}_{\alpha}+\mathrm{Q}_{\alpha}
\end{gathered}
$$

where $\overline{\mathrm{E}}$ and $\overline{\mathrm{B}}$ are the electric and magnetic fields, and subscripts $\alpha$ and $\beta$ represent either e or $i ; e_{\alpha}$ is the charge ( $e_{i} \equiv$ Ze, $e_{e} \equiv-$ e), $\overline{\bar{\Pi}}_{\alpha}$ the viscous stress tensor, $\vec{q}_{\alpha}$ the heat flux, $\bar{R}_{\alpha}\left(Q_{\alpha}\right)$ the force per unit volume on species $\alpha$ (the heat per unit volume generated in the gas of species $\alpha$ ) due to collisions 
with particles of the other species. These equations, together with the laws of Faraday and Ampère,

$$
\begin{gathered}
\partial \mathbf{B} / \partial \mathbf{t}=-\nabla \wedge \bar{E} \\
\varepsilon_{0} c^{2} \nabla \wedge \bar{B}=-e n \bar{u}
\end{gathered}
$$

and a single continuity equation,

$$
\left(\frac{\partial}{\partial t}+\bar{u}_{1} \cdot \nabla\right) n+n \nabla \cdot \bar{u}_{1}=0
$$

$(\nabla \cdot \mathrm{n} \overline{\mathrm{u}}=0$ being automatically satisfied), form a complete system for $\mathrm{n} \equiv \mathrm{n}_{\mathrm{e}}\left(\mathrm{n}_{\mathrm{i}} \because \mathrm{n} / \mathrm{Z}\right), \overline{\mathrm{u}}_{\mathrm{i}}, \overline{\mathrm{u}}\left(\overline{\mathrm{u}}_{\mathrm{e}} \equiv \overline{\mathrm{u}}_{\mathrm{j}}+\overline{\mathrm{u}}\right), \mathrm{T}_{\mathrm{e}}, \mathrm{T}_{\mathrm{i}}, \overline{\mathrm{E}}$, and $\bar{B} ; \epsilon_{0}$ is the permitivity of vacuum, $c$ is the speed of light. The left-hand side of Equation 1 for $\alpha=\mathrm{e}$, involving electron momentum, is naturally negligible $\left(\mathrm{m}_{e} \mathrm{u}_{\mathrm{e}}^{2} \& \mathrm{~T}_{e}\right)$.

Equations 1 and 2 are derived from the corresponding kinetic equation:

$$
\frac{\partial f_{\alpha}}{\partial t}+\bar{v} \cdot \nabla f_{\alpha}+\frac{e_{\alpha}}{m_{\alpha}}(\bar{E}+\bar{v} \wedge \bar{B}) \cdot \frac{\partial f_{\alpha}}{\partial \bar{v}}=c_{\alpha}
$$

In terms of $f_{\alpha}$ and the collision term $C_{\alpha}$, one has

$$
\begin{aligned}
& \overline{\overline{\mathrm{I}}}_{\alpha}=\int \mathrm{f}_{\alpha} \mathrm{d} \overline{\mathrm{v}}_{\alpha}\left[\left(\overline{\mathrm{v}}-\overline{\mathrm{u}}_{\alpha}\right)\left(\overline{\mathrm{v}}-\overline{\mathrm{u}}_{\alpha}\right)-\frac{1}{3} \overline{\mathrm{I}}\left|\overline{\mathrm{v}}-\overline{\mathrm{u}}_{\alpha}\right|^{2}\right] \\
& \overline{\mathrm{q}}_{\alpha}=\int \mathrm{f}_{\alpha} \mathrm{d} \overline{\mathrm{v}} \frac{1}{2} \mathrm{~m}_{\alpha}\left|\overline{\mathrm{v}}-\overline{\mathrm{u}}_{\alpha}\right|^{2}\left(\overline{\mathrm{v}}-\overline{\mathrm{u}}_{\alpha}\right) \\
& \overline{\mathrm{R}}_{\alpha}=\int \mathrm{m}_{\alpha}\left(\overline{\mathrm{v}}^{-} \overline{\mathrm{u}}_{\alpha}\right) \mathrm{c}_{\alpha} \mathrm{d} \overline{\mathrm{v}} \\
& \mathrm{Q}_{\alpha}=\int \frac{1}{2} \mathrm{~m}_{\alpha}\left|\overline{\mathrm{v}}-\overline{\mathrm{u}}_{\alpha}\right|^{2} \mathrm{c}_{\alpha} \mathrm{d} \overline{\mathrm{v}}
\end{aligned}
$$

with $\overline{\bar{I}}$ the unit tensor. These quantities must be obtained from a Chapman-Emskog solution of Equation $6 .^{5}$ From conservation of momentum and energy in collisions, one has, however, $-\overline{\mathrm{R}}_{\mathrm{i}}=\overline{\mathrm{R}}_{\mathrm{e}} \equiv \overline{\mathrm{R}}, \mathrm{Q}_{\mathrm{e}}+\overline{\mathrm{u}} \cdot \overline{\mathrm{R}}=-\mathrm{Q}_{\mathrm{i}} \equiv-\mathrm{Q}$.

In a monatomic gas, both viscous and thermal diffusivities are roughly given by the product of mean free path and thermal speed; their ratio, the Prandtl number, is near unity. A mixture of such gases has an effective Prandtl number around unity, also. A plasma is again quite different in this respect. Ion diffusivities are smaller than electron diffusivities by a factor of the order $\left(\mathrm{m}_{\mathrm{c}} / \mathrm{m}_{\mathrm{i}}\right)^{1 / 2}$. In addition, since electron momentum convection is itself negligible, one may also ignore the electron viscous tensor $\overline{\bar{\Pi}}_{e}$. The effective Prandtl number of a plasma is therefore very low, electron heat conduction being the dominant diffusion process. In fact, the structure, for instance, of a plasma shock may be entirely determined by the heat flow; when viscosity does count, its effects, due to ions, are limited to a thin sublayer within the overall shock structure. ${ }^{16,17}$ The case of the coronal plasma ablated from a target is, in this sense, quite similar. Here, we shall ignore $\overline{\bar{\Pi}} \alpha$ and $\bar{q}_{i}$ and just briefly comment on $\mathrm{Q}$ later. The right-hand side of Equation 2 is now $-\bar{\nabla} \cdot \bar{q}_{e}-\bar{R} \cdot \bar{u}-Q$ for electrons, and $Q$ for ions. ${ }^{18}$

From Equation 1, one obtains the total momentum equation:

$$
\frac{m_{1}}{\mathrm{z}_{1}} n\left(\frac{\theta}{\partial t}+\bar{u}_{1} \cdot \nabla\right) \bar{u}_{1}=-\nabla n\left(T_{t}+\frac{T_{1}}{\mathrm{z}_{1}}\right)-\operatorname{en} \overline{\mathrm{u}} \wedge \bar{B}
$$

which, with Equations 2 and 5, suffice to determine the flow under one-dimensional conditions, when the magnetic force vanishes. In general, however, Equations 3 and 4 , and the electron momentum equation:

$$
0=-\nabla\left(n T_{e}\right)-\operatorname{en}\left(\bar{E}+\bar{u}_{i} \wedge \bar{B}\right)+\bar{R}-\operatorname{en} \bar{u} \wedge \bar{B}
$$

are also needed. Note that if we write $\partial / \partial t+\overline{\mathrm{u}}_{\mathrm{i}} \cdot \nabla$ instead of $\partial / \partial \mathrm{t}+\overline{\mathbf{u}}_{\mathrm{e}} \cdot \nabla$ on the left of Equation 2 for electrons (as in Equations 5, 7, and 2 for ions) a term $-T_{e} n \bar{u} \cdot \nabla \ln \left(T_{e}^{3 / 2} / n\right)$ must be added to its right. Both $\bar{q}_{e}$ and $\bar{R}$ have components proportional to $\nabla \mathrm{T}_{\mathrm{e}}$ and $\overline{\mathrm{u}}$, given by Braginskii:

$$
\overline{\mathrm{R}}=\overline{\mathrm{R}}_{\mathrm{u}}+\overline{\mathrm{R}}_{\mathrm{T}} \quad \overline{\mathrm{q}}_{\mathrm{u}}=\overline{\mathrm{q}}_{\mathrm{g}}+\overline{\mathrm{q}}_{\mathrm{u}}
$$

$\overline{\mathrm{R}}_{\mathrm{T}}$ and $\overline{\mathrm{q}}_{\mathrm{u}}$ are thermoelectric effects.

While Braginskii's two-fluid description of transport is now widely used, it is still common to present results on plasma transport giving $\bar{u}$, or the current density - en $\bar{u}$, as a thermodynamic flux, in the language of the thermodynamics of irreversible processes, ${ }^{19}$ driven by thermodynamic forces $\nabla \mathrm{T}_{\mathrm{c}}$, $\overrightarrow{\mathrm{E}}+\overline{\mathrm{u}}_{\mathrm{i}} \wedge \overrightarrow{\mathrm{B}}$, and $\nabla \mathrm{n}$ (or $\nabla \mathrm{T}_{\mathrm{e}}, \overline{\mathrm{E}}$, and total pressure gradient $\nabla \mathrm{p}$, if $T_{i}=0, \vec{u}_{i}=0$ ). Essentially, however, this procedure mixes pure transport results (Equation 9), with a macroscopic balance equation (Equation 8) and leads to clumsy expressions. In addition, it may suggest that an electric field, and a density or pressure gradient, is incompatible with total equilibrium, and is necessarily an entropy source, which, of course, is not the case. On the other hand, there can be no total equilibrium if $\overline{\mathrm{R}} \neq 0$. Braginskii, moreover, cleverly turned around the role of $\overline{\mathrm{l}}$, considering it, together with $\nabla \mathrm{T}_{c}$, as a force driving fluxes $\overline{\mathrm{R}}$ and $\overline{\mathrm{q}}_{\mathrm{e}}$, the very quantities which, like $\overline{\bar{\Pi}}_{\alpha}, \overline{\mathrm{q}}_{\mathrm{i}}$, and $\mathrm{Q}$, had to be related to the fluid variables of the conservation equations. The entropy source in Equation 2 for electrons is then

$$
\begin{aligned}
& -\frac{\nabla \cdot \bar{q}_{e}+\bar{R} \cdot \bar{u}}{T_{e}} \equiv-\nabla \cdot\left(\frac{\bar{q}_{e}}{T_{e}}\right)+\frac{\bar{q}_{r} \cdot \nabla \ln T_{e}+\bar{R}_{u} \cdot \bar{u}}{T_{e}} \\
& +\frac{\bar{q}_{u} \cdot \nabla \ln T_{\theta}+\bar{R}_{T} \cdot \bar{u}}{T_{\theta}}
\end{aligned}
$$

(See Reference 13 for the contribution of $Q$ to the entropy growth.) The first term leads to the usual entropy flux at the boundaries of the plasma, and the last one vanishes as required 
by Onsager's principle. The middle term is the entropy source; if $\bar{u} \neq 0$, the entropy will indeed grow, irrespective of the values of $\nabla \mathrm{T}$, or $\overline{\mathrm{E}}$, or $\nabla \mathrm{n}$.

Although the case for using a two-fluid formulation in neutral-gas mixtures is weaker, the situation is not really different. In Equation 1, one uses $\bar{F}_{\alpha}$, instead of $e_{\alpha}\left(\bar{E}+\bar{u}_{\alpha} \wedge \bar{B}\right)$, for the external force on a particle of the $\alpha$-species $(\alpha, \beta=$ 1,2). Since $\bar{u}=\bar{u}_{1}-\bar{u}_{2}$ here is small compared with both $\bar{u}_{1}$ and $\bar{u}_{2}$, an expression for $\overline{\mathrm{R}}_{21}$, in addition to the equation for total momentum, may be obtained from Equation 1 :

$$
\left(\frac{1}{m_{1} n_{1}}+\frac{1}{m_{2} n_{2}}\right) \bar{R}_{21}=\frac{\vec{F}_{2}}{m_{2}}-\frac{\bar{F}_{1}}{m_{1}}-\frac{\nabla\left(n_{2} T\right)}{m_{2} n_{2}}+\frac{\nabla\left(n_{1} T\right)}{m_{1} n_{1}}
$$

From the kinetic equation, Chapman and Cowling ${ }^{20}$ find that a quantity, $\overline{\mathrm{d}}_{12}=p \overline{\mathrm{R}}_{21}$, has components proportional to $\overline{\mathrm{u}}$ and $\nabla \mathrm{T},{ }^{20}$ that is, they find $\overline{\mathrm{R}}_{21}=\overline{\mathrm{R}}_{\mathrm{u}}+\overline{\mathrm{R}}_{\mathrm{T}}$; when used in conjunction with Equation 10, this gives the ustal diffusion velocity, $\bar{u}$, in terms of gradients of $\mathrm{T}$, total pressure $\mathrm{p}$, and concentration, as well as the difference $\vec{F}_{2} / m_{2}-\vec{F}_{1} / m_{1}$.

To conclude this discussion, we note that in estimating the ion-to-electron diffusivity ratio we took $\mathrm{T}_{\mathrm{i}} / \mathrm{T}_{\mathrm{e}}, \mathrm{Z}$, and $\Omega \tau_{\mathrm{e}}(\Omega$ is the electron girofrequency, $\tau_{\mathrm{e}}$ is some characteristic collision time) to be of the order of unity. If, however, these quantities are large enough, ions could have a dominant tole.

\section{CLASSICAL RESULTS FOR A LORENTZIAN PLASMA}

We only need Equation 6 for electrons, so we start by recalling the Fokker-Planck collision term $\mathrm{C}_{\mathrm{e}}$, in the Landau form, ${ }^{21}$

$$
\begin{aligned}
& C_{e}=\sum_{g} C_{e s} \equiv \sum_{s} \frac{2 \pi \mu e^{2} \mathrm{e}^{2}}{m_{0}^{2}} \frac{\partial}{\partial \bar{v}} \\
& \cdot \int \bar{U} \cdot\left\{f_{s}\left(\bar{v}^{\prime}\right) \frac{\partial f_{e}}{\partial \bar{v}}-\frac{m_{e}}{m_{s}} f_{e}(\bar{v}) \frac{\partial f_{s}}{\partial \bar{v}^{\prime}}\right\} d \bar{v}^{\prime}
\end{aligned}
$$

Here $\Lambda$ is the Coulomb logarithm and

$$
\overline{\mathrm{U}}\left(\overline{\mathrm{v}}-\overline{\mathrm{v}}^{\prime}\right) \equiv \frac{\bar{I}\left|\overline{\mathrm{v}}-\overline{\mathrm{v}^{\prime}}\right|^{2}-\left(\overline{\mathrm{v}}-\overline{\mathrm{v}}^{\prime}\right)\left(\overline{\mathrm{v}}-\overline{\mathrm{v}}^{\prime}\right)}{|\overline{\mathrm{v}}-\overline{\mathrm{v}}|^{3}}
$$

An exactly equivalent result was given by Rosenbluth and coworkers. ${ }^{22}$ Integration by parts and use of $\partial \cdot \overline{\bar{U}} / \partial \bar{v}=$ $-\partial \cdot \overline{\mathrm{U}} / \partial \overline{\mathrm{v}}^{\top}$ leads to a convenient expression:

$$
\begin{aligned}
& \mathrm{C}_{\mathrm{n}}(\overline{\mathrm{v}})=\frac{2 \pi \Lambda \mathrm{e}^{2} \mathrm{e}^{2}}{\mathrm{~m}^{2}} \frac{\partial}{\partial \bar{v}}
\end{aligned}
$$

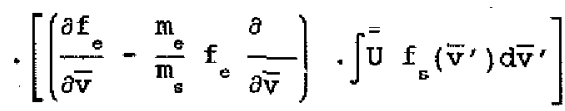

As usual, Equation 6 for $\mathrm{s}=\mathrm{e}$ is rewritten in the electron frame $\left(\bar{w} \equiv \bar{v}-\bar{u}_{e}\right)$ :

$$
\begin{gathered}
\frac{\partial f_{e}}{\partial t}+\left(\bar{u}_{o}+\bar{w}\right) \cdot v f_{a}-\left[\frac{e}{m_{e}}\left(\bar{E}+\bar{u}_{e} A \bar{B}\right)+\frac{\partial \overline{u_{e}}}{\partial t}+\left(\bar{u}_{e}+\bar{w}\right) \cdot \nabla \overline{u_{e}}\right] \cdot \frac{\partial f_{e}}{\partial \bar{w}} \\
=C_{e e}(\bar{w})+C_{e l}(\bar{w})+\bar{w} \cdot\left(\bar{\Omega}_{\Lambda} \frac{\partial f_{e}}{\partial \bar{w}}\right)
\end{gathered}
$$

where $\bar{\Omega} \equiv \mathrm{e} \overline{\mathrm{B}} / \mathrm{m}_{\mathrm{e}}$. In the classical limit both $\mathrm{C}_{\mathrm{e} i}$ and $\mathrm{C}_{\mathrm{ee}}$ are dominant against terms on the left of Equation 12, so that one may write

$$
f_{E}(\bar{w})=f_{H}(w)[1+\varphi(\bar{w})] \quad \varphi \text { smal } 1
$$

with $f_{M} \equiv n\left(m_{c} / 2 \pi T_{e}\right)^{3 / 2} \exp \left(-m_{e} w^{2} / 2 T_{e}\right)$, the local Maxwellian. Now one may set $f_{z}=f_{M}$ in the left-hand side of Equation 12. The standard procedure for a neutral gas would be next to use ideal gas conservation laws (here Equations 1 and 2 for $\alpha$ $=\mathrm{e}$, with $\overline{\bar{\Pi}}_{\mathrm{e}}, \overline{\mathrm{q}}_{\mathrm{e}}$, and $\mathrm{Q}_{\mathrm{e}}$ terms omitted, and the electron continuity equation) to transform that left-hand side to the form:

$$
\begin{aligned}
& f_{M} \frac{m_{e} \overline{W W}}{T_{e}}:\left(\nabla \bar{u}_{e}-\frac{1}{3} \overline{\bar{I}} \nabla, \bar{u}_{e}\right)+f_{H} \bar{W} \\
& \cdot\left[\frac{\bar{R}}{n T_{e}}+\left(\frac{m_{e} w^{2}}{2 T_{e}}-\frac{5}{2}\right) \nabla l n T_{e}\right]
\end{aligned}
$$

The first term would lead to the viscous tensor; the second one, larger by a factor $\left(T / m_{e} u_{e}^{2}\right)^{1 / 2}$, would yield $\bar{R}$ and $\bar{q}_{e}$ in terms of $\overline{\mathrm{u}}$ and $\nabla \mathrm{T}_{\mathrm{e}}$.

The viscous term that had to be dropped from, say, Equation 1 , is indeed smaller than the pressure term retained by a factor $\lambda_{\mathrm{T}} \mathrm{u}_{\mathrm{e}} / \mathrm{H}\left(\mathrm{T}_{\mathrm{e}} / \mathrm{m}_{\mathrm{e}}\right)^{1 / 2}$. However, dropping $\overline{\mathrm{q}}_{\mathrm{c}}$ from Equation 2 requires both $\mathrm{u}_{\mathrm{e}} \gg \mathrm{u}$ and $\mathrm{u}_{\mathrm{e}} \gg \lambda_{\mathrm{T}}\left(\mathrm{T}_{\mathrm{e}} / \mathrm{m}_{\mathrm{e}}{ }^{1 / 2}\right) / \mathrm{H}$. These are unnecessary restrictions. In fact, in laser fusion, heat conduction and energy convection are usually comparable in the region between the ablation and critical surfaces, thus requiring $u_{e}$ $\lambda_{\mathrm{T}}\left(\mathrm{T}_{e} / \mathrm{m}_{\mathrm{e}}\right)^{1 / 2} / \mathrm{H}$. ${ }^{18}$. Then, $\mathrm{Q}_{\mathrm{e}}$ must also be retained in Equation 2 for $\alpha=$ e. Since electron viscous effects are negligible anyway, the efficient procedure for the electron gas is just to ignore the entropy equation and, based on the inequality $\mathrm{u}_{\mathrm{e}} \ll$ $\left(\mathrm{T}_{\mathrm{e}} / \mathrm{m}_{\mathrm{e}}\right)^{1 / 2}$, reduce Equation 12 to the form:

$$
\begin{aligned}
& \bar{w} \cdot \nabla f_{e}-\frac{e}{m_{\theta}}\left(\overline{\mathrm{E}}+\bar{u}_{e} \wedge \overline{\mathrm{B}}\right) \cdot \frac{\partial f_{e}}{\partial \bar{w}}=C_{e s}(\bar{w}) \\
& +C_{e l}(\bar{w})+\bar{w} \cdot\left(\overline{\mathrm{w}} \Lambda \frac{\partial f_{e}}{\partial \bar{w}}\right)
\end{aligned}
$$


Using Equation 8 and $f_{e}=f_{M}$, the left-hand side of Equation 14 becomes

$$
\mathbf{f}_{H} \bar{w} \cdot\left[\frac{\overline{\mathrm{R}}}{\bar{n} \mathrm{~T}_{e}}+\left(\frac{\mathrm{m}_{e} \mathrm{w}^{2}}{2 \mathrm{~T}_{e}}-\frac{5}{2}\right) \nabla \ln \mathrm{T}_{e}\right]
$$

If terms of the order $\mathrm{m}_{\mathrm{e}} / \mathrm{m}_{\mathrm{i}}$ are neglected, Equation 11 for $\mathrm{C}_{\mathrm{ei}}$ can be greatly simplified. Take first the ion frame $\left(\bar{w}_{*} \equiv\right.$ $\left.\bar{v}-\bar{u}_{i}\right) ;$ using $\int f_{i} d \bar{W}_{*}=n / Z$ and $\int f_{i} \bar{W}_{*} d \bar{W}_{*}=0$, and expanding $\overline{\overline{\mathrm{U}}}\left(\overline{\mathrm{W}}_{*}-\overline{\mathrm{W}}_{*}^{\prime}\right)$ in powers of $\mathrm{W}^{\prime}{ }_{*} / \mathrm{W}_{*}-\left(\mathrm{m}_{\mathrm{e}} / \mathrm{m}_{\mathrm{j}}\right)^{1 / 2}$, one clearly has

$$
C_{e l}\left(\bar{w}_{*}\right)=\frac{2 \pi \Lambda e^{4} \mathrm{Zn}}{\mathrm{m}_{e}^{2}} \frac{\partial}{\partial \bar{w}_{*}} \cdot\left(\frac{\partial f_{e}}{\partial \bar{w}_{*}} \cdot \frac{\overline{\bar{I}_{*}^{2}} \bar{w}_{*} \bar{w}_{*} \bar{w}_{*}}{w_{*}^{3}}\right)
$$

Note that $\mathrm{C}_{\mathrm{ei}}$ now represents pure scattering (it will vanish if $f_{e}$ is isotropic); thus, to determine $Q$, one should retain the neglected terms of the order of $\mathrm{m}_{c} / \mathrm{m}_{\mathrm{i}}$, or use the ion kinetic equation. From Equation 13 and $\bar{w}_{*}-\bar{w}=\bar{u}$, with $\mathbf{u} \ll$ $\left(\mathrm{T}_{e} / \mathrm{m}_{\mathrm{e}}\right)^{1 / 2}$, we find

$$
f_{*}\left(\bar{w}_{*}\right)=f_{H}\left(w_{*}\right)\left[1+\varphi\left(\bar{w}_{*}\right)+m_{e} \bar{u} \cdot \bar{w}_{*} / T_{e}\right]
$$

Finally, using this $f_{e}\left(\bar{W}_{*}\right)$ in $C_{e j}\left(\bar{W}_{*}\right)$, and then setting $\bar{W}_{*} \simeq \bar{W}$, we obtain

$$
c_{e 1}(\bar{w})=c_{e i}^{\prime}\left(f_{i H} \varphi\right)-f_{H}(w) m_{e} \bar{u} \cdot \bar{w} / T_{e} \tau_{e I}(w)
$$

where, for $f_{e}(\bar{w})$ arbitrary,

$$
C_{e=1}^{\prime}\left(f_{e}\right) \equiv \frac{\partial}{\partial \bar{w}} \cdot \frac{\overline{\bar{t} w^{2}-\bar{w} w}}{2 \tau_{a}} \cdot \frac{\partial f}{\partial \bar{w}} \quad \tau_{e 1} \equiv \frac{m_{e}^{2} w^{3}}{4 \pi \Lambda e^{4} \mathrm{z} n}
$$

If, now, we take $\mathrm{Z}$ large, so that $\mathrm{C}_{\mathrm{e}}$ may be neglected against $\mathrm{C}_{\mathrm{e} i}$, Equation 14 becomes

$$
\begin{aligned}
& f_{M} \bar{w} \cdot \bar{c}(w)=C_{e 1}^{s}\left(f_{H} \varphi\right)+(\bar{w} \wedge \bar{\Omega}) \cdot \frac{\partial}{\partial \bar{W}}\left(f_{M} \varphi\right) \\
& \bar{c} \equiv \frac{\bar{R}}{n T_{e}}+\left(\frac{m_{e} w^{2}}{2 T_{e}}-\frac{5}{2}\right) \nabla \ln T_{e}+\frac{m_{e} \bar{u}}{T_{e} \tau_{e j}(w)}
\end{aligned}
$$

Trying a solution:

$$
\varphi=\bar{w} \cdot \bar{g}(w) / f_{M}
$$

in Equation 15 one immediately finds $C_{e i}^{\prime}=-\bar{w} \cdot \vec{g} \tau_{e l}$, and

$$
\bar{g}=-\tau_{e l} f_{K}\left[\bar{c}_{\|}+\frac{\bar{c}_{\perp}+c_{e l} \bar{\Omega}_{\Lambda} \bar{c}_{\perp}}{1+\left(\tau_{e l} \Omega\right)^{2}}\right]
$$

where $\bar{c}_{f}$ and $\bar{c}_{\perp-}$ are components parallel and perpendicular, respectively, to $\bar{B}$. Clearly, transport along $\bar{B}$ will be the same as in the absence of $\overline{\mathrm{B}}$.

The mean velocity of the electrons vanishes in their own frame, $\int f_{e}(\bar{w}) \bar{w} d \bar{w}=0$. This condition gives

$$
\begin{aligned}
& \int_{0}^{\infty} \tilde{\varepsilon}^{3} e^{-\tilde{\varepsilon}} \mathrm{d} \tilde{\varepsilon} \bar{c}_{\|}=0 \quad\left(\tilde{\varepsilon} \equiv m_{e} w^{2} / 2 \mathrm{~T}_{e}\right) \\
& \int_{0}^{m} \tilde{\varepsilon}^{3} e^{-\tilde{\varepsilon}} d \tilde{\varepsilon} \frac{\bar{c}_{\perp}+\Omega \tilde{\varepsilon}^{3 / 2} \bar{b} \wedge \bar{c}_{1}}{1+\Omega^{2} \tilde{\varepsilon}^{3}}=0
\end{aligned}
$$

where $\overline{\mathrm{b}} \equiv \overline{\mathrm{B}} / \mathrm{B}$ and $\bar{\Omega} \equiv 4 \Omega \tau_{\mathrm{e}} / 3 \pi^{t / 2}, \tau_{\mathrm{e}}$ being a convenient ion-electron collision time,

$$
\begin{aligned}
& \tau_{e} \equiv 3 \mathrm{~m}_{e}^{1 / 2} \mathrm{~T}_{e}^{3 / 2} / 4(2 \pi)^{1 / 2} \Lambda e^{4} \mathrm{z} \mathrm{n} \\
& =3 \pi^{1 / 2} \tau_{e 1}\left[\left(2 \mathrm{~T}_{e} / \mathrm{m}_{e}\right)^{1 / 2}\right] / 4
\end{aligned}
$$

Equation 19 yields

$$
\begin{aligned}
& \overline{\mathrm{R}}_{\|}=-\alpha_{0} \mathrm{~m}_{\mathrm{e}} n \overline{\mathbf{u}}_{\|} / \tau_{\mathrm{e}}-\beta_{0} n \nabla_{\|} \mathbf{T}_{\mathrm{e}} \\
& \alpha_{0}=3 \pi / 32 \quad \beta_{0}=3 / 2
\end{aligned}
$$

After a straightforward manipulation, Equation 20 gives

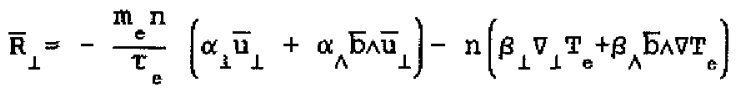

where

$\alpha_{\perp}(\Omega) \equiv \frac{9 \pi \mathrm{J}_{3} / 16}{\mathrm{~J}_{3}^{2}+\Omega^{2} \mathrm{~J}_{9 / 2}^{2}} \quad \alpha_{\Lambda}(\Omega) \equiv \frac{3 \pi^{1 / 2}}{4} \Omega \frac{\mathrm{J}_{3}^{2}-\mathrm{J}_{3 / 2} \mathrm{~J}_{9 / 2}}{\mathrm{~J}_{3}^{2}+\Omega^{2} \mathrm{~J}_{9 / 2}^{2}}$

$\beta_{\perp}(\Omega) \equiv \frac{\mathrm{J}_{4} \mathrm{~J}_{3}+\Omega^{2} \mathrm{~J}_{9 / 2} \mathrm{~J}_{11 / 2}}{\mathrm{~J}_{3}^{2}+\Omega^{2} \mathrm{~J}_{9 / 2}^{2}}-\frac{5}{2} \quad \beta_{\Lambda}(\Omega) \equiv \tilde{\Omega} \frac{\mathrm{J}_{3} \mathrm{~J}_{11 / 2}-\mathrm{J}_{4} \mathrm{~J}_{9 / 2}}{\mathrm{~J}_{3}^{2}+\Omega^{2} \mathrm{~J}_{9 / 2}^{2}}$

$J_{m}(\Omega) \equiv \int_{0}^{\infty} \frac{\tilde{\varepsilon}^{\mathrm{m}} e^{-\bar{\varepsilon}} d \tilde{\varepsilon}}{1+\tilde{\Omega}^{2} \tilde{\varepsilon}^{3}}$

From $\bar{q} \equiv \int 1 / 2 m_{e} w^{2} \overrightarrow{w f}_{e}(\bar{w}) d \bar{w}$, we find

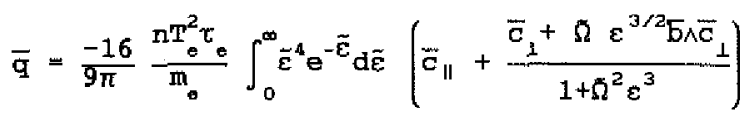

For $\overline{\mathrm{q}}_{\mathrm{i}}$, using Equation 21, we find in particular

$$
\overline{\mathrm{q}}_{\|}=-\gamma_{0} \frac{\mathrm{nT} \tau_{e}}{\mathrm{~m}_{\theta}} \nabla_{\|} \mathrm{T}_{e}+\beta_{0} \mathrm{nT} \overline{\mathrm{u}}_{\|} \quad \gamma_{0} \equiv 128 / 3 \pi
$$


Again, using Equation 22, there results

$$
\begin{aligned}
& \bar{q}_{\perp}=-\frac{\pi \mathrm{T}_{e} \bar{\tau}_{e}}{\mathrm{~m}_{e}}\left(\gamma_{\perp} \nabla_{\perp} \mathrm{T}_{e}+\gamma_{\Lambda} \bar{b}_{\Lambda} \nabla_{\perp} \mathrm{T}_{e}\right) \\
& +\mathrm{IT}_{e}\left(\beta_{\perp} \overline{\mathrm{u}}_{\perp}+\beta_{\lambda} \mathrm{b}_{\Lambda} \overline{\mathrm{u}}_{\perp}\right)
\end{aligned}
$$

We will not pause to give $\gamma_{\perp}(\tilde{\Omega}), \gamma_{\wedge}(\tilde{\Omega})$. Epperlein ${ }^{14}$ has graph ically compared all six perpendicular coefficients with Braginskii's approximate results.

One can also use the ion frame to determine $\bar{R}$ and $\bar{q}_{e}$. We rewrite Equation 14 by setting $\overline{\mathbf{u}}_{\mathrm{e}} \rightarrow \overline{\mathbf{u}}_{\mathrm{i}}, \overline{\mathrm{w}} \rightarrow \overline{\mathrm{w}}^{*}, \mathrm{C}_{\mathrm{e}}(\overline{\mathrm{w}}) \rightarrow$ $\mathrm{C}^{\prime}{ }_{\text {ei }}\left(\overline{\mathrm{W}}_{*}\right)$ as obtained earlier, and arrive at Equation 15 with $\overline{\mathrm{W}} \rightarrow \overline{\mathrm{w}}_{*}, \varphi \rightarrow \varphi_{*}, \overline{\mathrm{c}} \rightarrow \overline{\mathrm{c}}_{*}=\overline{\mathrm{c}}-\mathrm{m}_{\mathrm{e}}\left(\overline{\mathrm{u}}-\tau_{\mathrm{e}} \bar{\Omega} \wedge \overline{\mathrm{u}}\right) / \tau_{\mathrm{ei}} \mathrm{T}_{\mathrm{e}}$. Its solution, similar to Equation 18 , naturally recovers the relation $\varphi_{*}=\varphi+m_{e} \bar{u} \cdot \bar{w} / T_{e}$. Using $n \bar{u}=\int f_{e}\left(\bar{W}_{*}\right) \bar{w}_{*} d \bar{w}_{*}$ and $\overline{\mathrm{q}}=\int 1 / 2 \mathrm{~m}_{\mathrm{e}}\left(\overline{\mathrm{w}}_{*}-\overline{\mathrm{u}}\right)^{2}\left(\overline{\mathrm{w}}_{*}-\overline{\mathrm{u}}_{\mathrm{e}} \mathrm{f}_{\mathrm{e}}\left(\overline{\mathrm{w}}_{*}\right) \mathrm{d} \overline{\mathrm{w}}_{*}=\int 1 / 2\right.$ $\mathrm{m}_{\mathrm{e}} \mathrm{W}_{*}^{2} \overline{\mathrm{W}}_{*} \mathrm{f}_{\mathrm{e}}\left(\overline{\mathrm{W}}_{*}\right) \mathrm{d} \overline{\mathrm{W}}_{*}-5 / 2 \mathrm{nu} \mathrm{T}_{e}$, Equations 21 to 24 follow.

\section{NONCLASSICAL HEAT FLUX}

$$
\text { For } \begin{aligned}
\bar{u}=0, \bar{B} & =0, \text { Equations } 16 \text { to } 18 \text { and } 21 \text { give } \\
\varphi & =-\tau_{e 1} \bar{w} \cdot \bar{c}-\lambda_{e 1}(w) / H
\end{aligned}
$$

where $\lambda_{\mathrm{ei}} \equiv 2 \mathrm{w} \tau_{\mathrm{ei}} \propto \mathrm{w}^{4}$ is the ion-electron mean free path, $\lambda_{\mathrm{T}}$ being its thermal value. When $\lambda_{\mathrm{T}} / \mathrm{H}$ grows to a value of the order of unity, classical transport should fail: we then have $\varphi$ $=0(1)$ at thermal speeds, the use of $f_{M}$ on the left of Equation 14 being no longer valid. Further, from Equation 23, we have

$$
q_{e}=q_{T}-\lambda_{T} n T_{e}^{3 / 2} / m_{e}^{1 / 2} H
$$

the maximum heat flux that can be carried by the electron population should be of the order $n \mathrm{~T}_{\mathrm{e}}\left(\mathrm{T}_{\mathrm{e}} / \mathrm{m}_{\mathrm{e}}\right)^{1 / 2}$, the so-called free-streaming value, and this is achieved for $\lambda_{\mathrm{T}} / \mathrm{H} \sim 1$.

Usually a flux limiter $f$ is introduced into numerical codes simulating experiments, in order to avoid, in a crude way, unphysically large heat fluxes in steep thermal fronts. The procedure is to take $q_{\mathrm{T}}$ as either the mininum or the harmonic mean of the free-streaming $\left(\mathrm{fn}_{\mathrm{s}}^{3 / 2} / \mathrm{m}_{\mathrm{v}}^{1 / 2}\right.$ ) and classical expressions. Results are affected by the procedure chosen; further, there is no definite value for the limiter. Apparently, however, f should be about unity, so that if $\lambda_{\mathrm{T}}>\mathrm{H}, \mathrm{q}_{\mathrm{T}}$ is reduced below its classical value; in a sense $\varphi$ is reduced. Typically a limiter $\mathrm{f} \simeq 0.6\left(3 / 2^{3 / 2} \pi^{1 / 2}\right)$ is taken from an estimate of the maximum flux carried by a Maxwellian, ${ }^{23}$ but the electron population may be far from Maxwellian for the conditions of interest. Values as low as 0.2 to 0.3 have been considered as an effect of the self-consistent electric field. ${ }^{24}$

In laser fusion, $H$ is often much larger than $\lambda_{\mathrm{T}}$ : for a characteristic speed $\mathrm{u}_{\mathrm{e}} \sim \mathrm{u}_{\mathrm{i}} \sim\left(\mathrm{ZT}_{\mathrm{e}} / \mathrm{m}_{\mathrm{i}}\right)^{1 / 2}$ (Section II), and from a balance of conduction and convection in the overdense plasma, $\mathrm{u}_{\mathrm{e}} \rightarrow \lambda_{\mathrm{T}}\left(\mathrm{T}_{\mathrm{e}} / \mathrm{m}_{\mathrm{e}}\right)^{1 / 2} / \mathrm{H}$ (Section III), we have

$$
H / \lambda_{T}-\left(m_{i} / 2 m_{e}\right)^{1 / 2}-60
$$

One might thus expect classical transport results to generally hold in the plasma blowing off a laser target. In the last 15 years, however, there has been experimental evidence for severe flux inhibition, that is, for the need to use substantially lower limiters. This means that classical results fail at large $\mathrm{H} / \lambda_{\mathrm{T}}$, a fact that requires an explanation.

The evidence can be very indirect, as from implosion experiments in which the neutron yield, the implosion time, or the X-ray image of the imploding target are determined. More direct evidence comes from the X-ray image of the hot plasma escaping the target; the current (vs. time) to a charge collector, which provides a spectrum of fast and slow ions; or the burnthrough of the heat front through a layered target, determined from line emission by a signature substratum (plastic or beryllium, over glass or aluminum). The case for strong inhibition was first substantiated in 1975 , by Malone et al. ${ }^{25}$ who analyzed simulations and experiments at large intensities and long wavelengths $(1.06$ to $10 \mu \mathrm{m})$. The lack, by the codes, of models for suprathermal electrons, and even radiation pressure effects, was later criticized. ${ }^{26,27}$ The evidence has also been reviewed by Kruer, ${ }^{28}$ Duderstadt, ${ }^{29}$ Ahlstrom, ${ }^{30} \mathrm{Max}^{31}$ and Rosen, ${ }^{32}$ and later by Delettrez ${ }^{24}$ and Kruer. ${ }^{33}$

Experiments with plane (usually disk) targets have suggested $f$ values ranging from 0.01 to $0.04 .^{34}$ Experiments with spherical targets, starting in 1982, have generally indicated a weaker inhibition, limiters roughly ranging from $0.03-0.06$ to $0.1 .^{35}$ Quite recent results, however, have emphasized that often different values are needed for numerical simulations to fit data on different physical magnitudes (say a temperature, or a mechanical time for the expansion); ${ }^{36}$ or that there is simply no single $f$ value that fits the experiments, ${ }^{37}$ Nonetheless, analyses of the plasma flow suggest that, on the whole, there is some dependence of flux inhibition on the geometry, which is plane, one-dimensional for short pulses or large targets and focal spots, and nearly spherical or hemispherical in opposite conditions. ${ }^{38}$

There have been multiple attempts to justify low limiters and establish transport models for use in simulation codes. It has been noticed that, as shown by Equation 18, a large magnetic field would greatly reduce the flux across it and, further, that according to Equations 3, 4, 8, and 21, a field will necessarily develop if $\nabla \mathrm{T}_{\mathrm{e}} \wedge \nabla_{\mathrm{n}} \neq 0 .{ }^{39}$ Although magnetic generation will not occur in perfect spherical geometry, smallscale inhomogeneities could produce a complex field topology ${ }^{40}$ However, full, economic handling of magnetic effects in transport is hardly possible in design codes.

Ion-sound turbulence has also been considered for flux inhibition, ${ }^{41} \mathrm{~A}$ decrease in ion-electron collision time would reduce $\varphi$, and thus the heat flux. Since $\tau_{e \mathrm{i}}^{-1} \propto \mathrm{n}_{\mathrm{i}} Z^{2}=\mathrm{nZ}$, the 
collision time for $n / Z$ ions of charge $Z$ is shoiter than that corresponding to $n$ ions of unit charge; this is because charges within an ion will scatter electrons coherently. Similarly, any low-frequency instability that enhances ion-density fluctuations will reduce the flux, through scattering of electrons off bunches of ions. It has been noticed that the ion-acoustic mode of a distribution function $\mathrm{f}_{\mathrm{M}}(1+\varphi)$ carrying a heat flux becomes Vlasov-unstable (Landau damping becoming negative) when the plasma profile steepens. Estimated levels of turbulence appear, however, too low to be effective. ${ }^{42}$

It was later realized that the strong energy dependence of plasma mean free paths, characteristic of a Coulomb cross section, might explain flux inhibition through a failure of classical transport theory that appears when main-body electrons are still highly collisional. ${ }^{27}$ For plane, one-dimensional geometry with neither $\overline{\mathrm{B}}$ nor $\overline{\mathrm{u}}$, and no turbulence, Equations 16 to 18 and 21 give

$$
\varphi=-\tau_{e t}(\tilde{\varepsilon}-4) \bar{w} \cdot \nabla I n T_{e}
$$

The integral for the heat flux now becomes

$$
q_{T} \propto \int_{0}^{\infty} \tilde{\varepsilon}^{4}(\tilde{\varepsilon}-4) e^{-\tilde{\varepsilon}} \mathrm{d} \bar{\varepsilon}
$$

The integrand has a maximum at $\tilde{\epsilon}=\tilde{\epsilon}^{*} \simeq 6,5$, so that electrons contributing most to the heat flux lie in the tail of the distribution function. Since $w \tau_{\mathrm{ej}} \propto \tilde{\epsilon}^{2}$, one could have both $\varphi$ small at thermal energies, $\tilde{\epsilon} \sim 1$, and $\varphi \sim 1$ at the energies of interest in transport, $\bar{\epsilon}-\tilde{\epsilon}^{*}$.

A variety of theoretical analyses and numerical calculations have dealt with this issue. Shvarts et al. ${ }^{43}$ noticed that for $\varphi \sim 1$ classical theory would make $f_{\mathrm{e}}$ negative at some range of velocity angles; to avoid this, they introduced an ad hoc limitation on $\varphi$. Further, with $\varphi \sim 1$, the isotropic part of the distribution function, $f_{0}$, need not be Maxwellian; Shkarofsky computed $q_{T}$ when $f_{o}$ is the sum of hot and cold populations. ${ }^{44}$ Bernstein, ${ }^{45}$ Gurevich and Istomin, ${ }^{46}$ and Krasheninnikov ${ }^{47}$ have considered solutions of the kinetic equation that distinguish different energy ranges. Considering a distribution function $f_{e}$ with a rich angular structure, Kishimoto and Mima arrived at Burnett-type transport results. ${ }^{48}$ Both Grad's 13-moment method $^{49}$ and a modified moment method ${ }^{50}$ have been tried without success. Numerical calculations of Monte Carlo type ${ }^{51}$ and numerical solutions of the Fokker-Planck equation ${ }^{52}$ have been obtained for both a plasma lying between plates and a steadily flowing plasma, simulating ablation. The Fokker-Plank results suggest a broad range of f-values centered around 0.1 .

A self-consistent transport model for Lorentzian plasmas was given by Albritton et al. ${ }^{53}$ If $Z$ is large, $f_{z}$ will fail to be Maxwellian while still being isotropic. For $\bar{\epsilon}^{*}$ electrons we thus write

$$
f_{e}(\bar{w})=f_{0}(w)[1+\varphi(\vec{w})] \quad \varphi \operatorname{small}
$$

with $\mathrm{f}_{\circ} \neq \mathrm{f}_{\mathrm{M}}$ in general; at thermal energies, Equation 13 will be assumed to hold. Consider, then, Equation 14 with $\bar{u} \equiv 0$, $\overline{\mathrm{B}} \equiv 0$. To dominant terms we may set $\mathrm{f}_{\mathrm{e}}=\mathrm{f}_{\mathrm{o}}$ in the left-hand side, and drop $\mathrm{C}_{\mathrm{ee}}$, to obtain

$$
\bar{W} \cdot\left(\nabla f_{a}-\frac{e \bar{E}}{m_{e} w} \frac{\partial f_{0}}{\partial W}\right)=C_{e 1}^{\prime}\left(f_{0} \varphi\right)
$$

Since $f_{o}$ is yet unknown, it is worth simplifying this equation by writing $f_{0}(\bar{r}, w)$ as $f_{0}\left(\bar{r}, \epsilon=1 / 2 m_{e} w^{2}-e \Psi\right)$, with $\bar{E} \equiv$ $-\nabla \Psi$ :

$$
\bar{w} \cdot \nabla f_{0}=C_{e 1}^{\prime}\left(f_{0} \varphi\right)
$$

Now the local Maxwellian takes the form $\mathrm{f}_{\mathrm{M}} \equiv \mathrm{n}\left(\mathrm{m}_{\mathrm{e}} / 2 \pi \mathrm{T}_{\mathrm{e}}\right)^{3 / 2}$ $\exp \left[-(\epsilon+\mathrm{e} \Psi) / \mathrm{T}_{\mathrm{e}}\right]$. Following Equation 17 , we try $\varphi=\overline{\mathrm{w}}$. $\overline{\mathrm{g}}(\mathrm{w}) / \mathrm{f}_{o}$ and find $\mathrm{C}_{\mathrm{ei}}^{\prime}=-\overline{\mathrm{w}} \cdot \overline{\mathrm{g}} / \tau_{\mathrm{ei}}$ and

$$
\bar{g}=-\tau_{e 1} \nabla f_{0}
$$

If $f_{o} \rightarrow f_{M}$, one uses Equations 8 and 16 and recovers Equation 18 with $\overline{\mathrm{u}}=0, \overline{\mathrm{B}}=0$.

To determine $f_{o}$, we average Equation 14 over velocity angles; $f_{v}$ on the left, and $C_{e i}^{\prime}$, give no contribution. We find

$$
-\frac{1}{3} w \nabla \cdot\left(\tau_{e i} w \nabla f_{0}\right)=C_{e e}\left(f_{0}, f_{0}\right)
$$

Since Equation 8 gives e $\Psi \sim \mathrm{T}_{e}$, we omitted in Equation 25 a tertn $\left(2 \tau_{e i} \mathrm{e} \overline{\mathrm{E}} / 3 \mathrm{~m}_{\mathrm{e}}\right) \cdot \nabla \mathrm{f}_{\mathrm{o}}$, which is small by a factor $2 \mathrm{e} \Psi / \mathrm{m}_{\mathrm{e}} \mathrm{w}^{2}$ $-1 / \bar{\epsilon}^{*}$, or $15 \%$. In handling Equation 25 we may similarly set $1 / 2 \mathrm{~m}_{\mathrm{e}} \mathrm{w}^{2}=\epsilon+\mathrm{e} \Psi=\epsilon$, when appearing in powers (but not in an exponential like the Maxwellian!). Note that the collision term itself has only logarithmic accuracy, the Coulomb logarithm being less than 10 for the high densities of interest here.

For self-collisions, Equation 11 becomes

$$
C_{e e}=\frac{2 \pi \Lambda e^{b}}{m_{e}^{2}}\left[\frac{\partial^{2} f}{\partial \bar{w} \partial w_{w}}: \frac{\partial^{2}}{\partial \bar{w} \partial \bar{w}} \int\left|\bar{w}-\bar{w}^{\prime}\right| f_{e}\left(\bar{w}^{\prime}\right) d \bar{w}^{\prime}+8 \pi f_{e}^{2}\right]
$$

where we set $f_{e} \approx f_{0}$. The last term is negligible for our tail electrons. Thermal speeds are dominant in the integral, so we write $f_{\mathrm{o}}\left(\mathrm{w}^{\prime}\right) \simeq \mathrm{f}_{M}\left(\mathrm{w}^{\prime}\right)$ and expand $\left|\overline{\mathrm{w}}-\overline{\mathrm{w}}^{\prime}\right|$ for small $\mathrm{w}^{\prime} / \mathrm{w}$. Only even powers contribute; we retain two terms, neglecting fourth and higher powers:

$$
C_{e e} \simeq \frac{m_{e} w^{2}}{\tau_{e l} z} \frac{\partial}{\partial \varepsilon}\left(f_{0}+T_{e} \frac{\partial f_{0}}{\partial \varepsilon}\right)
$$

Since $T_{e} / \epsilon$ is small, the last term should be neglected, for consistency, if $f_{o}$ followed a power law, but not if it were an 
exponential like the Maxwellian; actually, $\mathrm{C}_{\mathrm{ee}}$ above will vanish if $f_{o}=f_{M}$. Here, one makes a crucial ansatz:

$$
\left|T_{O} \partial\left(f_{0}-f_{M}\right) / \partial E\right| \ll\left|f_{D}-f_{H}\right|
$$

and thus obtains

$$
C_{e e}=\frac{\mathbb{m e}_{e} w^{2}}{Z \tau_{\theta 1}}\left(\frac{\partial \mathbf{f}_{0}}{\partial \varepsilon}-\frac{\partial f_{H}}{\partial \varepsilon}\right)
$$

Using Equation $27^{\prime}$ in Equation 25, taking the heat flux along $\mathrm{x}$ and defining $\mathrm{d} \xi=\left(3 / 8 \mathrm{Z}_{\mathrm{i}}\right)^{1 / 2} \mathrm{~m}_{\mathrm{e}}^{2} \mathrm{w}^{3} \tau_{\mathrm{es}}^{-1} \mathrm{dx}$, we arrive at an equation for $\mathrm{f}_{o}$,

$$
\frac{\partial \mathbf{f}_{0}}{\partial \varepsilon}+\varepsilon^{3} \frac{\partial^{2} \mathbf{f}_{0}}{\partial \xi^{2}}=\frac{\partial \mathbf{f}_{H}}{\partial \varepsilon}
$$

This is a "heat diffusion" equation with $-\epsilon^{4 / 4}$ as a time-like variable. For an infinite plasma, with $f_{o}$ vanishing in the "remote past" $(\epsilon \rightarrow \infty)$, the solution is clearly ${ }^{53}$

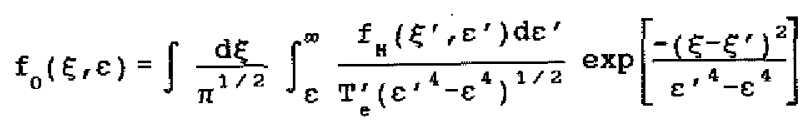

which can be shown to satisfy the ansatz; here $\mathrm{T}_{e}^{\prime} \equiv \mathrm{T}_{e}\left(\xi^{\prime}\right)$. Note that the non-Maxwellian population for suprathermal energy $\epsilon$ at a position $\xi$ arises from a Maxwellian source of electrons at $\epsilon^{\prime}>\epsilon$, which lost energy while randon walking from a neighbor position $\xi^{\prime}$ !

For a profile with comparable high and low temperatures $T_{h}, T_{1}$, or if $T_{e}-T_{h} \geqslant T_{l}$, we will have $\epsilon \geqslant T_{e}^{\prime}$. Introducing a second ansatz, $\mathrm{Hd} \xi / \mathrm{dx} \ll \epsilon^{5 / 2} / \mathrm{T}_{\mathrm{e}}^{\prime 1 / 2}$, only values of $\epsilon^{\prime}$ close to $\epsilon$ are found to contribute to the $\epsilon^{\prime}$-integral, which can then be carried out: ${ }^{54}$

$$
f_{0}(\xi, \varepsilon)=\int \frac{f_{H}\left(\xi^{\prime}, \varepsilon\right) d \xi^{\prime}}{2\left(\varepsilon^{3} T_{e}^{\prime}\right)^{1 / 2}} \exp \left[\frac{-\left|\xi^{\prime}-\xi^{\prime}\right|}{\left(\varepsilon^{3} T_{e}^{\prime}\right)^{1 / 2}}\right]
$$

The parameter range of interest is clearly $\Delta \xi \sim$ $2 \epsilon^{3 / 2} T_{e}^{1 / 2}$, which is equivalent to

$$
\mathrm{H} \sim \mathrm{z}^{1 / 2} \overrightarrow{\mathrm{\varepsilon}}^{*^{3 / 2}} \lambda_{\mathrm{T}}
$$

The second ansatz follows immediately from Equation 32 and $\mathrm{T}_{\mathrm{e}}^{\prime} \ll \epsilon$. Note also that the range in Equation 32 agrees $\mathrm{nu}-$ merically with the characteristic distance between the ablation and critical surfaces, $H \sim\left(m_{i} / Z m_{e}\right)^{1 / 2} \lambda_{r}$, previously found. Again, for $T_{e}-T_{1} \ll T_{h}$ and Equation 32 satisfied at the top of the profile, electrons carrying the heat from the top should be fully collisional at the typical bottom density $n_{1} \sim n_{h} T_{k} / T_{1}$.
For thermal energies, $\epsilon \sim T_{e}$, and $H$ satisfying Equation 32, Equation 31 is a convolution of $f_{M}\left(\xi^{\prime}, \epsilon\right)$ and a $\delta$-function, giving $f_{o}=f_{M}$. For a larger scale length, Equation 31 would yield $f_{o}=f_{M}$ at the suprathermal energies of interest, $\epsilon \sim$ $6.5 \mathrm{~T}_{e}$, recovering the classical result from

$$
\begin{aligned}
& 0=\int \bar{w} d \bar{w}\left(-\tau_{e i} \bar{w} \cdot \nabla f_{0}\right) \\
& \bar{q}_{e}=\int \frac{1}{2} m_{e} w^{2} \bar{w} d \bar{w}\left(-\tau_{e 1} \bar{w} \cdot \nabla f_{0}\right)
\end{aligned}
$$

with $\mathrm{f}_{\mathrm{o}}=\mathrm{f}_{\mathrm{M}}$ and $\nabla \ln \mathrm{f}_{\mathrm{M}}=\nabla \ln \left(\mathrm{n} / \mathrm{T}_{\mathrm{e}}^{3 / 2}\right)+\mathrm{e} \overline{\mathrm{E}} / \mathrm{T}_{\mathrm{e}}+(\epsilon+$ $\left.\mathrm{e}^{\mathrm{r}}\right) \mathrm{T}_{\mathrm{e}}^{-1} \nabla \ln \mathrm{T}_{\mathrm{e}}$, and using Equation $8, \overline{\mathrm{q}}_{\mathrm{T}}=-\left(128 \mathrm{nT}_{\mathrm{e}} \tau_{\mathrm{e}}{ }^{\prime}\right.$ $\left.3 \pi \mathrm{m}_{\mathrm{c}}\right) \nabla \mathrm{T}_{\mathrm{e}}$ follows. At the other extreme, when $\mathrm{H}$ is much less than Equation 32, Equation 31 should ultimately break down. Since the model allows $f_{n}-f_{M}$ to be comparable to $f_{M}$, Equation 28 may be rewritten as

$$
\left|\partial\left(f_{0}-f_{M}\right) / \partial \varepsilon\right| \ll\left|\partial f_{M} / \partial \varepsilon\right|
$$

this is compatible with $\left|f_{o}-f_{M}\right| \sim f_{M}$ only for a narrow range $\Delta \epsilon \sim T_{e}$ (in the tail of the distribution) which, nonetheless, can include the electrons carrying most of the flux. If $\mathbf{f}_{M}$ changes in a "distance" $\Delta \xi \ll\left(\epsilon^{3} \mathrm{~T}_{\mathrm{e}}\right)^{1 / 2}$, the solution to Equation 29 lags well behind, and Equation 35 will fail. Prasad and Kershaw $^{55}$ have illustrated the failure of Equation 29 for some extreme and peculiar profiles. Ramíre ${ }^{56}$ has used the model with full hydrodynamics to study the flow off a target and found it valid up to a laser intensity one order of magnitude above that for which classical transport fails. Unfortunately, the model will not signal its failure when used in a code.

Introducing Equation 31 and Equations 33 and 34 yields two coupled equations for $\mathrm{q}_{\mathrm{T}}$ and an auxiliary field $\mathrm{E}_{\mathrm{nl}}$ :

$\left\{0, \mathrm{q}_{\mathrm{T}}\right\}=\int \frac{-\left\{1, \mathrm{~T}_{\mathrm{a}}^{\prime}\right\} \mathrm{n}^{\prime} \mathrm{d} \mathrm{X}^{\prime}}{4 \pi\left(3 \mathrm{~m}_{\mathrm{e}} \mathrm{Z}_{1} \mathrm{~T}_{\mathrm{e}}^{\prime}\right)^{1 / 2}}\left[\left\{\mathrm{Y}^{*}, K^{*}\right\} \frac{\mathrm{dT}^{\prime}}{\mathrm{dx^{ \prime }}}+\left\{J^{*}, L^{*}\right\} \in E_{\mathrm{n} 1}^{\prime}\right]$

where $\mathrm{eE}_{\mathrm{nl}}=\mathrm{eE}+\mathrm{T}_{\mathrm{e}} \mathrm{dlnn} / \mathrm{dx}-5 / 2 \mathrm{dT}_{\mathrm{e}} / \mathrm{dx}$, and the kernels $\mathrm{I}^{*}, \mathrm{~J}^{*}, \mathrm{~K}^{*}$, and $\mathrm{L}^{*}$ are functions of $\theta \equiv\left|\xi-\xi^{\prime}\right| / \mathrm{T}^{12}$ given in terms of one single integral:

$$
J^{*}(\theta)=8 \pi^{1 / 2} \int_{0}^{\infty} s^{3 / 2} \exp \left(-s-\theta / s^{3 / 2}\right) d s
$$

$I^{*}=3 J^{*}-2 \theta \mathrm{dJ}^{*} / \mathrm{d} \theta, \mathrm{L}^{*}=\left(3 \mathrm{I}^{*}+\mathrm{J}^{*}\right) / 4, \mathrm{~K}^{*}=4 \mathrm{~L}^{*}-2 \theta \mathrm{dL}^{*} /$ $\mathrm{d} \theta$. If Equation 30 is used insteady of Equation 31 , the resulting kernels $I, J, K, L$ involve two single and two double unconnected integrals. ${ }^{53,55}$ All kernels have a width $\Delta \theta \sim 15$; requiring $\mathrm{H}(\mathrm{d} \xi / \mathrm{dx}) / \mathrm{T}^{\prime 2} \sim \Delta \theta$ leads to Equation 32 . For $H$ large, only the complete integrals of the kernels are needed; they are equal for the starred and unstarred expressions, e.g., $\int_{0}^{\infty}$ ( $\left.\mathrm{I}^{*}-\mathrm{I}\right) \mathrm{d} \theta=0$, both recovering the classical results. For small $H$, only the values at $\theta=0$ are needed; they are slightly higher 
for the starred expressions $\left(\mathrm{I}^{*} / \mathrm{I}=\mathrm{J}^{*} / \mathrm{J}=1.18, \mathrm{~K}^{*} / \mathrm{K}=\mathrm{L}^{*} / \mathrm{L}\right.$ $\simeq 1.12$ at $\theta=0$ ), so Equation 31 gives a heat flux $12 \%$ above that from Equation 30 , the difference falling within the asymptotic accuracy of the model.

If $\mathbf{u} \neq 0$ one uses the ion-frame to solve Equation 14 . Since only the isotropic part of $\mathrm{C}_{\mathrm{ec}}$ is needed, Equations 29 to 31 are unmodified while the left-hand sides of Equations 33 and 34 now read nu and $\mathrm{q}_{e}+5 / 2 \mathrm{nT}_{e} \mathrm{u}$, respectively. Also, using Equation 8 , one has $\mathrm{eE}_{\mathrm{nl}}=\mathrm{R} / \mathrm{n}-7 / 2 \mathrm{dT}_{\mathrm{e}} / \mathrm{dx}$; to express $R$ and $q_{e}$ in terms of $d T_{e} / d x$ and $u$, it suffices to make the change $\mathrm{I}^{*} \mathrm{dT}_{\mathrm{e}}^{\prime} / \mathrm{dx}^{\prime}+\mathrm{J}^{*} \mathrm{eE}_{\mathrm{nl}}^{\prime} \rightarrow\left(\mathrm{I}^{*}-7 / 2 \mathrm{~J}^{*}\right) \mathrm{dT}_{\mathrm{e}}^{\prime} / \mathrm{dx}^{\prime}+\mathrm{J}^{*} \mathrm{R}^{\prime} / \mathrm{n}^{\prime}$, and similarly for $\mathrm{K}^{*}, \mathrm{~L}^{*}$, in Equation 36. As $\mathrm{H}$ decreases from large to small values, $R_{\mathrm{T}}$ changes sign varying from its classical value $-3 / 2 \mathrm{nT}_{e} / \mathrm{dx}$ to $1 / 2 \mathrm{ndT} / \mathrm{dx}$. At short scale lengths, Onsager's principle, requiring $\mathrm{q}_{\mathrm{u}} \mathrm{dln} \mathrm{T}_{\mathrm{e}} / \mathrm{dx}=\mathrm{R}_{\mathrm{T}} \mathrm{u}$, is not satisfied, in general. ${ }^{54}$

It can be shown that

$$
\frac{\int_{0}^{\infty} K^{*} d \theta}{\int_{0}^{\infty} L^{*} d \theta}-\frac{\int_{0}^{\infty} I^{*} d \theta}{\int_{0}^{\infty} J^{*} d \theta}=\frac{K^{*}(0)}{I^{*}(0)}-\frac{I^{*}(0)}{J^{*}(0)}=1
$$

which also applies, of course, to the unstarred kernels. Consequently, the formula ${ }^{54}$

$$
q_{T}=\int \frac{-n^{\prime} T_{e}^{\prime} d x^{\prime}}{4 \pi\left(3 m_{e} Z_{1} T_{B}^{\prime}\right)^{1 / 2}} \quad L^{\prime \prime} \frac{d T_{e}^{\prime}}{d x}
$$

agrees exactly with Equation 36 for both Iong (or classical) and short scale limit profiles, and it should be a convenient approximation for intermediate cases. Luciani and co-workers first developed, in a series of papers, ${ }^{57}$ a nonlocal formalism for the heat flux. In particular, they obtained a convolution formula similar to Equation 37, with a simple exponential fitted to numerical simulations and the classical limit as kernel, and derived Equation 31. Lindman and Swartz have given an alternative convolution formula. ${ }^{58}$ Holstein and Decoster ${ }^{59}$ have discussed and compared nonlocal models; see also Reference 54 for a discussion on models.

\section{EFFECTS OF ELECTRON-ELECTRON SCATTERING}

For $Z$ of the order of unity, self-collisions contribute to the nonisotropic part of $\mathrm{C}_{\mathrm{e}}$. Classical results cannot then be given in closed form, as in Equations 21 to 24; some numerical approximation, usually in the form of a broken Laguerre expansion for $f_{c}-f_{M}$, must be introduced. Here, we derive approximate analytical results by using one basic point of the model leading to Equation 29, namely, that a solution for $f_{e}-f_{M}$ is needed only at suprathermal energies. ${ }^{60}$
The starting equation is Equation 15 , with a term $\mathrm{C}_{\mathrm{ee}}$ added to its right-hand side. In Equation 26 the last term is exponentially small and, as previously, $\left|\bar{w}-\bar{w}^{\prime}\right|$ in the integral is expanded for small $w^{\prime} / w$. Now $f_{e}=f_{M}(1+\varphi)$ and both even and odd powers give contributions; however, since $\int f_{e} \bar{w} d \bar{w}=$ 0 , we obtain the same two-term expansion for $\mathrm{C}_{\mathrm{ce}}$ if third and higher powers are neglected. Using Equation 17, one has

$$
c_{e e} \simeq \frac{-\bar{w}}{z \tau_{e i}} \cdot\left[\bar{g}\left(1-\frac{1}{2 \tilde{\varepsilon}}\right)-\frac{\partial}{\partial w}\left(w \bar{g}+\frac{\partial}{\partial \tilde{\varepsilon}} w \bar{g}\right)\right]
$$

Equation 15, modified, now gives

$$
f_{M} \bar{c}=-\frac{z+1}{z \tau_{e i}} \bar{g}+\bar{\Omega} \wedge \bar{g}+\frac{1}{z \tau_{e i}} \frac{\partial}{\partial w}\left(w \bar{g}+\frac{\partial w \bar{g}}{\partial \tilde{\varepsilon}}\right)
$$

A term $1 / 2 \bar{E}(1+Z)$ was dropped against unity; for $Z \rightarrow \infty$, one recovers Equation 18. We shall just consider Equation 39 in the absence of $\overline{\mathrm{B}}$ (or along $\overline{\mathrm{B}}$ ). Then defining

$$
h=3 \pi^{2} T_{e} w g / z m_{e} n \tau_{e}, \quad 2 N \equiv z+1
$$

we finally arrive at

$$
\frac{\mathrm{d}^{2} h}{d \bar{\varepsilon}^{2}}+\frac{d h}{d \tilde{\varepsilon}}-\frac{N}{\tilde{\varepsilon}} h=\tilde{E} e^{-\tilde{E}} c(\tilde{\varepsilon})
$$

The homogeneous part of Equation 40 is the equation for an associated Laguerre polynomial, $\mathrm{L}_{\mathrm{N}}^{(-1)}(-\bar{\epsilon})$, which we just write $\mathrm{L}_{\mathbf{N}}(\tilde{\epsilon})$,

$$
\mathrm{L}_{\mathrm{N}}(\bar{\varepsilon}) \equiv \frac{\tilde{\varepsilon}}{\mathrm{N} J} e^{-\tilde{\varepsilon}} \frac{\mathrm{d}^{\mathrm{N}}}{\mathrm{d}^{\mathrm{N}}}\left(e^{\tilde{\varepsilon}} \tilde{\varepsilon}^{\mathrm{N}-1}\right)
$$

normalized so that $\mathrm{L}_{\mathrm{N}} / \bar{\epsilon} \rightarrow 1$ as $\bar{\epsilon} \rightarrow 0$; we have $\mathrm{L}_{1}=\tilde{\epsilon}, \mathrm{L}_{2}$ $=\tilde{\epsilon}+\bar{\epsilon}^{2} / 2, L_{3}=\tilde{\epsilon}+\tilde{\epsilon}^{2}+\tilde{\epsilon}^{3} / 6, \ldots$ A convenient second solution is $\mathrm{L}_{\mathrm{N}} \int_{\bar{\epsilon}}^{\infty} \mathrm{e}^{-\bar{\epsilon}^{\prime}} \mathrm{d} \bar{\epsilon}^{\prime} / \mathrm{L}_{\mathrm{N}}^{2}\left(\bar{\epsilon}^{\prime}\right)$. Using the boundary condition $h \rightarrow 0$ as $\tilde{\epsilon} \rightarrow \infty$, the full solution to Equation 40 reads

$$
h=L_{N} \int_{\tilde{\varepsilon}}^{\infty} \frac{e^{-\tilde{\varepsilon}^{*}} d \tilde{\varepsilon}^{\prime}}{L_{N}^{2}\left(\tilde{E}^{\prime}\right)}\left[A-\phi\left(\tilde{\varepsilon}^{\prime}\right)\right]
$$

with $\mathrm{A}$ being a constant and

$$
\phi \equiv \int_{0}^{\bar{\varepsilon}} \tilde{\varepsilon} L^{\prime} L_{N}\left(\bar{\varepsilon}^{\prime}\right) \text { c }\left(\tilde{\varepsilon}^{\prime}\right) d \tilde{\varepsilon}^{\prime}
$$


$\mathrm{R}$ must have the form of Equation 21, with $\alpha_{0}, \beta_{\mathrm{o}}$ unknown functions of $\mathrm{Z}$; thus,

$$
c=\left(\tilde{\varepsilon}-\frac{5}{2}-\beta_{0}\right) \frac{d n^{T} \mathrm{~T}_{e}}{d x}+\left(\frac{3 \pi^{1 / 2}}{4 \tilde{\varepsilon}^{3 / 2}}-\alpha_{0}\right) \frac{m_{e} u}{T_{e} \tau_{e}}
$$

Condition $\int f_{\mathrm{e}} \overline{\mathrm{w}} \mathrm{d} \overline{\mathrm{w}}=0$ now reads $\int_{0}^{\infty} \bar{\epsilon}(\bar{\epsilon}) \mathrm{d} \tilde{\boldsymbol{\epsilon}}=0$, giving

$$
A=\frac{f_{0}^{\infty} \phi F_{1} e^{-\tilde{\varepsilon}} d \tilde{\varepsilon} / L_{\mathrm{N}}^{2}}{f_{0}^{\infty} F_{1} e^{-\tilde{\varepsilon}_{\mathrm{E}}} \mathrm{d} / \mathrm{L}_{\mathrm{N}}^{2}}
$$

where $F_{1}=\int_{o}^{\bar{\epsilon}} \bar{\epsilon}^{\prime} L_{N}\left(\bar{\epsilon}^{\prime}\right) d \bar{\epsilon}^{\prime}$. From $\bar{q}=\int 1 / 2 \mathrm{~m}_{e} \mathrm{w}^{2} \overline{\mathrm{w}} \mathrm{d} \overline{\mathrm{w}} \mathrm{f}_{\mathrm{e}}$, with $\bar{q}$ taking the form of Equation 23, we obtain

$$
\beta_{0} \frac{m_{e} u}{T_{v} \tau_{c}}-\gamma_{0} \frac{d l n T_{c}}{d x}=\frac{8 Z}{9 \pi} \int_{0}^{\infty} \frac{F_{z} e^{-\bar{\varepsilon}}}{L_{\mathrm{N}}{ }^{2}}(A-\phi) d \tilde{\varepsilon}
$$

where $\mathrm{F}_{2}=\int_{0}^{\dot{\varepsilon}} \tilde{\boldsymbol{\epsilon}}^{\prime 2} \mathrm{~L}_{\mathrm{N}}\left(\tilde{\boldsymbol{\epsilon}}^{\prime}\right) \mathrm{d} \tilde{\mathrm{\epsilon}}^{\prime}$. From Equations 41 to 44 , two relations, between $\alpha_{o}$ and $\beta_{o}$, and $\beta_{o}$ and $\gamma_{o}$, respectively, are derived,

No boundary condition for $\mathrm{h}$ at $\bar{\epsilon}=0$ has been used, because our solution is only valid at large $\ddot{\epsilon}$. We now just require that the solution for $\mathrm{h}$ makes our simplified form for $\mathrm{C}_{\mathrm{ee}}$ satisfy the condition of $\int \mathrm{m}_{\mathrm{e}} \overline{\mathrm{w}} \mathrm{C}_{\mathrm{ee}} \mathrm{d} \overline{\mathrm{w}}=0$, as it should. Our approximations had lost this as a general property of $\mathrm{C}_{\mathrm{ee}}$ (note that at large $\mathrm{Z}$ the nonisotropic part of $\mathrm{C}_{\mathrm{es}}$ had negligible effects). Since $\bar{R}=\int m_{e} \bar{w} C_{r} d \bar{w}$, as defined in Section II, we may write the condition as $\overline{\mathrm{R}}=\int \mathrm{m}_{\mathrm{e}} \overline{\mathrm{w}} \mathrm{C}_{\mathrm{e} i} \mathrm{~d} \overline{\mathrm{w}}$. We then obtain

$$
\left(1-\alpha_{0}\right) \frac{m_{e} u}{T_{e} \tau_{0}}-\beta_{0} \frac{d I n T_{t}}{d x}=\frac{-2 Z_{1}}{3 \pi^{1 / 2}} \int_{0}^{\infty} \frac{d \tilde{\varepsilon} F_{3} e^{-\tilde{\varepsilon}}}{I_{W}^{2}}(A-\phi)
$$

where $F_{3}=\int_{\mathrm{v}}^{\epsilon} \mathrm{L}_{\mathrm{N}}\left(\tilde{\epsilon}^{\prime}\right) \mathrm{d} \tilde{\epsilon}^{\prime} / \tilde{\epsilon}^{\prime 1 / 2}$, yielding two equations for $\alpha_{v}$ and $\beta_{\mathrm{o}}$. That means that our approximation leads to different values for $\beta_{\mathrm{o}}$ in $\overline{\mathrm{R}}_{\mathrm{T}}$ and $\overline{\mathrm{q}}_{\mathrm{u}}$. We impose Onsager's principle by taking some mean value from the two equations for $\beta_{\circ}$.

Since errors from our approximations on $\mathrm{C}_{\mathrm{ce}}$ will decrease as $\mathrm{Z}$ increases and $\mathrm{C}_{\mathrm{ee}} / \mathrm{C}_{\mathrm{e}}$ decreases, we check our procedure with the worst case, $Z=1(N=1)$, which is also the simplest to fully carry out. From Equation 44, we obtain ${ }^{60}$

$$
\frac{3 \pi}{4} \gamma_{0}(1)=10-4 \beta_{0}(1) \quad \frac{3 \pi}{4} \beta_{0}(1)=4 \alpha_{0}(1)-\frac{15 \pi}{64}
$$

and similarly, from Equation 45,

$$
\alpha_{0}(1)=\frac{15 \pi}{154}+\frac{2}{11} \quad \beta_{0}(1)=\frac{185}{154}-\frac{15}{88}
$$

We thus arrive at $\alpha_{0}(1)=0.49$, which compares well with the "exact" numerical result, 0.51 , in Reference 13. The two solutions for $\beta_{0}(1)$ are then 0.52 and 1.03 ; their geometric and arithmetic means are 0.73 and 0.77 , respectively, to be compared with the exact value of $0.71 .{ }^{13}$ Finally, using either mean in the equation for $\gamma_{0}(1)$, we obtain 3.01 and 2.93, compared with the exact value, 3.16 , given by Braginskii. The errors, 3 to $8 \%$, do not exceed the inaccuracy in the Coulomb logarithm.

The following point is important: if one makes the ansatz that the last term in Equation 39, and therefore the first term in the left-hand side of Equation 40, is negligible, in an approximation similar to that leading to Equation $27^{\prime}$ (that is, one recalls that $\tilde{\epsilon}$ is large and assumes that $\mathrm{h}$ behaves like a power rather than as an exponential), the resulting solution is found not to verify the ansatz. Furthermore, that solution does not satisfy the condition $\int \mathrm{m}_{e} \bar{w}_{\mathrm{ee}} \mathrm{d} \overline{\mathrm{w}}=0$. Note also that Equation 40 suggests that electron self-scattering must be already considered at values $\mathrm{N} \sim 6.5$ or $\mathrm{Z} \sim 10$.

Thus, an extension of the nonlocal model of Reference 53 to include self-scattering is required for values $Z \leq 10$. That extension faces two difficulties. First, there is now no large parameter allowing for an asymptotic expansion, with $f_{e}$ not nearly Maxwellian and yet near-isotropic. In this respect, however, there is evidence suggesting that even at low Z the electron population becomes isotropic sensibly faster than Maxwellian. ${ }^{57}$

Thus, using Equation $13^{\prime}$ with $\varphi=\bar{w} \cdot \bar{g}(w) / f_{o}$ and changing to variables $\overline{\mathrm{r}}, \varepsilon=1 / 2 \mathrm{~m}_{\mathrm{e}} \mathrm{W}^{2}-\mathrm{e} \Psi$, the nonisotropic part of Equation 14 , for $\overline{\mathrm{u}} \equiv 0, \overline{\mathrm{B}} \equiv 0$, reads

$$
\vec{w}_{.} \nabla f_{0}=C_{01}^{\prime}\left(f_{0} \varphi\right)+C_{e e}
$$

with $\mathrm{C}_{\mathrm{ee}}$ given by Equation 38, or, for gradients along $\mathrm{x}$,

$$
\frac{\partial f_{o}}{\partial x}=-\frac{z+1}{z \tau_{o 1}} g+\frac{m_{o} w}{z t_{o 1}} \frac{\partial}{\partial \varepsilon}\left(w g+T_{e} \frac{\partial}{\partial \varepsilon} w g\right)
$$

The isotropic part of Equation 14 now reads

$$
\frac{w}{3} \frac{\partial}{\partial x}(w g)=c_{t e t}\left(f_{o}, f_{0}\right)
$$

with $\mathrm{C}_{\mathrm{ee}}$ given by Equation $27^{\prime}$.

The other difficulty is the last term in Equation 46, which made Equation 40 second order. The evidence from our analysis of the classical case suggests that this term should not be dropped, apparently precluding the attainment of $q_{T}$ in closed form. If dropped, however, one arrives at a hyperbolic equation for $f_{0}$,

$$
\frac{\partial f_{0}}{\partial \varepsilon}+\frac{Z}{1+Z} \varepsilon^{3} \frac{\partial^{2} f_{0}}{\partial \xi^{2}}=\frac{\partial f_{H}}{\partial \varepsilon}-\frac{2 \varepsilon^{2}}{1+Z} \frac{\partial}{\partial \varepsilon}\left[\frac{1}{\varepsilon} \frac{\partial}{\partial \varepsilon}\left(f_{0}-f_{M}\right)\right]
$$


with $\xi$ as defined in Section V. Minotti and Ferro Fontán ${ }^{61}$ have neglected the term in question, and solved a similar equation for $\mathrm{g}$ in terms of Bessel functions of the order $|Z-3| / 8$ and large argument. Using asymptomatic expansions, for fixed order $[\mathrm{Z}=0(1)]$, they found an explicit formula for the heat flux, in very good agreement with the experimental, nonclassical data of Reference 37 for $Z=1$. They could not, however, recover the high $Z$ results of Section IV or the classical results at low Z. Recently, complete agreement and generality have been obtained by using asymptotic expansions for Bessel functions of large argument and order and simplifying the last term in Equation 46 , by following the procedure that led to Equation 27. ${ }^{1,62}$

\section{REFERENCES}

1. Sanz, J., Liñân, A., Rodrígıez, M., and Sanmartín, J. R., Quasisteady expansion of plasma ablated from laser-irradiated pellets, Phys. Fluids, 24, 2098, 1981 .

2. Shkarofsky, I. P., Johnston, T. W., and Bachinsky, M. A., The Particle Kinetics of Plasmas, Addison-Wesley, Reading, MA, 1966.

3. Hochstim, A. R. and Massel, G. A., Calculation of transport coefficients in ionized gases, in Kinetic Processes in Gases and Plosmas, Hochstim, A. R., Ed., Academic Press, New York, 1969, 141.

4. Epperlein, E. M. and Haines, M. G., Plasma transport coefficients in a magnetic field by direct numerical solution of the Fokker-Planck equation, Phys. Fluids, 29, 1029, 1986.

5. Chapman, S. and Cowling, T. G., The Mathematical Theory of NonUniform Gases, Cambridge University Press, Cambridge, 1970.

6. Spitzer, L. and Härm, R., Transport phenomena in a completely ionized gas, Phys. Rev., 89, 977, 1953.

7. Burgers, J. M., Statistical plasma mechanics, in Plasma Dynamics, Clauser, F. H., Ed., Addison-Wesley, Reading, MA, 1960, 119.

8. Herdan, R. and Liley, $\mathbf{B}_{+}$, Dynamical equations and transport relationships for a thermal plasma, Rev. Mod. Phys., 32, 731, 1960.

9. Thompson, W. H., An Introduction to Plasma Physics, Addison-Wes ley, Reading, MA, 1964, 225 and 244.

10. Robinson, B. B. and Bernstein, I. B., A variational description of transport phenomena in a plasma, Ann. Phys., 18, 110, 1962.

11. Cowling, T. G., The electrical conductivity of an ionized gas in a magnetic field, with applications to the solar atmosphere and the ionosphere, Proc. R. Soc. London Ser. A, 183, 453, 1945.

12. Landshoff, R., Transport phenomena in a completely ionized gas in presence of a magnetic field, Phys. Rev., 76, 904, 1949.

13. Braginskit, S. I., Transport processes in a plasma, in Reviews of Plasma Physics, Vol. 1, Consultant Bureau, New York, 1965, 205.

14. Epperlein, E. M., The accuracy of Braginskii's transport coefficients for a Lorentz plasma, J. Phys. D, 17, 1823, 1984.

15. Lee, Y. T. and More, R. M., An electron conductivity model for dense plasmas, Phys. Fluids, 27, 1273, 1984.

16. Jaffrin, M. Y. and Probstein, R. F., Structure of a plasma shock wave, Phys. Fluids, 7, 1658, 1964.

17. Zeldovich, Ya. B. and Raizer, Yu. P., Physics of Shock Waves and High-Temperature Hydrodynamic Phenomena, Vol. 2, Academic Press, New York, 1967, chap. 7.

18. Sanmartín, J. R., Coronal fluid-dynamics in laser fusion, Laser Part. Beams, 7, 219, 1989.
19. de Groot, S. R. and Mazur, P., Non Equilibrium Thernodynamics, North-Holland, Amsterdam, 1962.

20. Chapman, S. and Cowling, T. G., The Mathematical Theory of NonUniform Gases, Cambridge University Press, Cambridge, 1970, chaps. 6 and 8 .

21. Landau, $\mathbf{L}$. D., The transport equation in the case of Coulomb interactions, in Collected Papers of L. D. Landaw, terHaar, D., Ed., Gordon and Breach, New York, 1967, 163.

22. Rosenbluth, M. N., MacDonald, W. M., and Judd, D. L., FokkerPlanck equation for an inverse-square law, Phys. Rev+ 107, 1, 1957.

23. Mason, R. J. and Morse, R. L., Hydrodynamics and burn of optimally imploded deuterium-tritium spheres, Phys. Fluids, 18, 814, 1975.

24. Delettrez, J., Thermal electron transport in direct-drive laser fusion, Can. J. Phys., 64, 932, 1986.

25. Malone, R. C., McCrory, R. L., and Morse, R. L., Indications of strongly flux-limited electron themal conduction in laser-target experiments, Phys, Rev, Lett, 34, 721, 1975.

26. Gitomer, S. J. and Henderson, D. H., Re-examination of strongly flux-limited thermal conduction in laser produced plasmas, Phys. Fluids, $22,365,1979$

27. Gray, D. R. and Kilkenny, J., The measurement of ion acoustic turbulence and reduced thermal conductivity caused by a large temperature gradient in a laser heated plasma, Plasma Phys., 22, 81, 1980.

28. Kruer, W. L., Electron-cnergy transport in laser-produced plasmas, Comments Plasma Phys., 5, 69, 1979.

29. Duderstadt, J. J., Inertial Confinement Fusion, John Wiley \& Sons, New York, 1982.

30. Ahlstrom, H. G., Laser fusion experiments at Lawrence Livermore Laboratory, in Les Houches, 1990, Balian, R. and Adams, J. C., Eds., Elsevier-North-Holland, New York, 1982, 1.

31. Max, C. E., Physics of the coronal plasma in laser fusion targets, in Les Houches, 1980, Balian, R. and Adams, J. C., Eds., ElsevierNorth-Holland, New York, 1982, 301.

32. Rosen, M. D., Evidence of a laser intensity threshold for the onset of inhibited electron transport, Comments Plasma Phys. Controlled Fu. sion, 8, 165, 1984.

33. Kruer, W. L,, The Physics of Laser Plasma Interactions, AddisonWesley, Reading, MA, 1988, chap. 12.

34. Young, F. C., Whitlock, R. R., Decoste, R., Ripin, B. H., Nagel, D. J., Stamper, J. A., McMahon, J. M., and Bodner, S. E., Laserproduced-plasma energy transport through plastic films, Appl. Phys. Lett, 30, 45, 1977; Yaakobi, B., Boehly, T., Bourke, P, Conturie, Y., Craxton, R. S., Delettrez, J., Torsyth, J. M., Frankel, K. D., Goldmar, L. M., McCrory, R. L., Richardson, M. C., Seka, W., Shvarts, D., and Soures, J. M., Characteristics of target interaction with high power UV laser radiation, Opt. Communt, 39, 175, 1981.; Mead, W. C., Campbell, E. M., Kruer, W. L., Turner, R. E., Hotcher, C. W., Bailey, D. S., Lee, P. H. Y., Foster, J., Tirsell, K. G., Pruett, B., Holmes, N. C., Traimor, J. J., Stradling, G. L., Lasinsky, B. F., Max, C. E., and Ze, F., Characteristics of lateral and axial transport in laser irradiations of layered-disk targets at 1.06 $\mu \mathrm{m}$ and $0.35 \mathrm{~km}$ wavelengths, Phys. Fluids, 27, 1301, 1984.

35. Goldsack, T. J., Kilkenny, J. D., MacGowan, B. J., Cunningham, P. F., Lewis, C. L. S., Key, M. H., and Rumsby, P. T., Evidence for large heat fluxes from the mass ablation rate of laser-irradiated spherical targets, Phys, Fluids, 25, 1634, 1982; fechner, W. B., Shepard, C. L., Busch, G. E., Schroeder, R. J., and Tarvin, J. A., Analysis of plasma density profiles and thermal transport in laserirradiated spherical targets, $P$ hys. Fluids, 27, 1552, 1984; Jaanimagi, P. A., Delettrez, J., Henke, H. L., and Richardson, M. C., Temporal dependence of the mass-ablation rate in UV-laser irradiated spherical targets, Phys. Rev. A, 34, 1322, 1986. 
36. Drake, R. P, Phillion, D. W., Estabrook, K., Turner, R. E., Kauffman, R. L., and Campbell, E. M., Hydrodynamic expansion of exploding-foil targets irradiated by $0.53 \mu \mathrm{m}$ laser light, Phys. Fluids $B, 1,1089,1989$.

37. Rogers, J. H., De Groot, J. S., Abou-Assaleh, Z., Matte, J. P. Johnston, T. W., and Rosen, M. D., Electron heat transport in a steep temperature gradient, Phys. Fluids B, 1, 741, 1989.

38. Sanz, J. and Sanmartin, J. R., Ion charge number and flux saturation effects in the corona of a laser-irradiated pellet, Phys. Fluids, 26, 3361, 1983; Ramis, R., and Sanmartin, J. R., Electron temperature versus laser intensity times wavelength squared: a comparison of theory and experiments, Nucl. Fusion, 23, 739, 1983.

39. Craxton, K. S. and Haines, M. G., Hot spots in laser plasmas, $P$ hys. Rev. Lett., 35, 1336, 1975.

40. Haines, M. G., Magnetic field generation in laser fusion and lotelectron transport, Can. J. Phys., 64, 912, 1986.

41. Manheimer, W. M., Energy flux limitation by ion acoustic turbulence in laser fusion schemes, Phys. Fluids, 20, 265, 1977.

42. Lindman, E. L., Absorption and transport in laser plasmas, J. Phys. (Paris) Colloq, 38 (6), 9, 1977.

43. Shvarts, D., Delettrez, J., McCrory, R. L., and Verdon, C. P. Self-consistent reduction of the Spitzer-Härm electron thermal heat flux in steep temperature gradients in laser-produced plasmas, Phys. Rev. Lett., 47, 247, 1981

44. Shkarofsky, I. P., Heat transport with cold and hot electrons allowing for flux limitation by Shvart's method, Phys. Fluids, 26, 3131, 1983.

45. Bernstein, I. B., Electron distribution functions and quasistatic electromagnetic fields in laser-pellet plasmas, Phys. Fluids, 20, 577, 1977.

46. Gurevich, A. Y. and Istomin, Ya. N., Thermal runaway and convective heat transport by fast electrons in a plasma, Sov. Phys. JETP, $50,470,1979$.

47. Krasheninnikov, S. I., Superthermal particles and electron thermal conductivity, Sov. Phys. JETP, 67, 2483, 1988.

48. Kishimoto, Y. and Mima, K., An extension of Spitzer-Härm theory on thermal transport to steep temperature gradient case. I. General formulation, J. Phys. Soc. Jpn, 52, 3389, 1983.

49. Moses, G. A. and Duderstadt, J. J., Improved treatment of electron themal conduction in plasma hydrodynamics calculations, Phys. Fluids, 20, 762, 1977; Djebar, H. K. and Moses, G. A., Analytical approach to the problem of nonlinear thermat conduction in laser-produced plasmas, Phys. Fluids, 28, 1460, 1985.
50. Clause, J. P. and Balescu, R., A nonlinear approach to the kinetic theory of heat conductivity in a plasma, Plasma Phys., 24, 1429, 1982.

51. Mason, R. J., Monte Carlo hybrid modeling of electron transport in laser produced plasmas, Phys. Fluids, 23, 2204, 1980; Mason, R. J, Apparent and real thermal inhibition in laser-produced plasmas, Phys. Rev. Lett., 47, 652, 1981; Khan, S. A. and Ronglein, T. J., Thermal heat flux for arbitrary collisionality, Phys. Fluids, 24, 1442, 1981.

52. Bell, A. R., Evans, R. G., and Nicholas, D. J., Electron energy transport in steep temperahure gradients in laser-produced plasmas, Phys. Rev. Lett., 46, 243, 1981; Matte, J. P. and Virmont, J., Electron heat transport down steep temperature gradients, Phys. Rev. Leth., 49, 1936, 1982; Bell, A. R., Non-Spitzer heat flow in a steadily ablating laser-produced plasma, Phys. Fluids, 28, 2007, 1985.

53. Albritton, J. R., Williams, E. A., Bernstein, L. B., and Swartz, K. P., Nonlocal electron heat transport by not quite Maxwell-Boltz. mann distributions, Phys. Rev. Lett., 57, 1887, 1986.

54. Sanmartín, J. R., Kamírez, J, and Fernández-Feria, R., Non-local electron heat flux revisited, Phys. Fluids B, 2, 2519, 1990.

55. Prasad M. K. and Kershaw, D. S., Nonviability of some non-local electron heat transport modeling, phys. Fluids B, 1, 2430, 1989.

56. Ramírez, J., Conducción Electuónica no Clásica en Plasmas Producidos por Luz Laser, Ph.D. thesis, Universidad Politécnica de Madrid, 1990.

57. Luciani, J. F., Mora, P., and Virmont, J., Nonlocal heat transport due to steep temperature gradients, Phys. Rev. Lett., 51, 1664, 1983; Luciani, J. F., Mora, P., and Pellat, R., Quasistatic heat front and delocalized heat flux, Phys. Fluids, 28, 835, 1985; Luciani, J. F. and Mora, P., Resummation methods of the Chapman-Emskog expansion for a strongly inhomogeneous plasma, J. Stat. Phys., 43, 281, 1986.

58. Lindman, E. L. and Swartz, K., Analytical studies of hot electron transport, Phys. Fluids, 29, 2657, 1986.

59. Holstein, P. A. and Decoster, A., Comparison between two models of nonlocal heat flux in laser-generated plasma, J. Appl. Phys., 62, $3592,1987$.

60. Sanmartín, J. R., Nonlocal electron heat-flux, in I0th European School of Plasma Physics, Tsindsadze, N., Ed., World Scientific, Singapore, 1991, 405.

61. Minotti, F. and Ferro-Fontán, C., Nonlocal heat transport in plasmas down steep temperature gradients, Phys. Fluids B, 2, 1725, 1990.

62. Sanmartín, J. R., Ramirez, J., Fernández-Feria, R., and Minotti, F., Selfconsistent nonlocal electron heat flux at arbitrary ion charge number, Phys. Fluids $B$, in press. 